\title{
2019 novel coronavirus (COVID-19) pneumonia complications: the importance of lung ultrasound
}

\author{
Letizia Consoli ${ }^{1}$. Vittorio Bendotti ${ }^{1}$ Sara Cicchinelli ${ }^{1,2} \cdot$ Federico Gaioni $^{1} \cdot$ Paola Prandolini ${ }^{3} \cdot$ Monica Bettonagli $^{1}$. \\ Paolo Terragnoli ${ }^{1}$
}

Received: 22 April 2020 / Accepted: 10 June 2020 / Published online: 19 June 2020

(c) Società Italiana di Ultrasonologia in Medicina e Biologia (SIUMB) 2020

\begin{abstract}
In December 2019, a novel coronavirus (SARS-Cov-2) was first reported in Wuhan, China, and rapidly spread around the world, leading to an international emerging public health emergency. As reported from Chinese experiences, approximately $20 \%$ of patients had a severe course, requiring intensive care, with an overall case fatality rate of $2.3 \%$. In diagnosis, chest computed tomography most commonly showed ground-glass opacity with or without consolidative patterns.

Herein, we report a case of a patient affected by COVID-19 pneumonia referred in the emergency department of our institution on April 4, 2020, with peculiar lung ultrasound findings.
\end{abstract}

Keywords Covid-19 $\cdot$ Pneumonia $\cdot$ Pneumothorax $\cdot$ Lung ultrasound

\section{Introduction}

The outbreak of an atypical pneumonia was first reported in Wuhan, the capital city of Hubei province in China, in December 2019 [1]. In January 2020, Chinese scientists isolated a novel coronavirus from patients affected by viral pneumonia, denominated severe acute respiratory syndrome coronavirus 2 (SARS-COV-2), and in February 2020, the World Health Organization designated as COVID-19 the coronavirus disease caused by SARS-COV-2.

As indicated in a report from the Chinese Center for Disease Control and Prevention on 44,500 SARS-COV-2 patients, severe respiratory symptoms were found in $14 \%$ of cases, characterized by dyspnea, hypoxia, or $>50 \%$ lung involvement on imaging. Five percent of the patients were critical (respiratory failure, shock, or multiorgan system dysfunction). In this study, all deaths occurred among patients with critical illness, and the overall case fatality rate was

Letizia Consoli

letizia.consoli@poliambulanza.it

1 Department of Emergency Medicine, Fondazione Poliambulanza, Brescia, Italy

2 Department of Emergency Medicine, Fondazione Policlinico A. Gemelli IRCCS, Rome, Italy

3 Department of Radiology and Medical Imaging, Fondazione Poliambulanza, Brescia, Italy
2.3\%. The case fatality rate among patients with critical disease was $49 \%$ [2]. The most common complications observed in severe cases included acute respiratory distress syndrome and respiratory failure, while less common complications included secondary infection, acute cardiac injury, hypoxic encephalopathy, acute kidney injury, shock, and acute liver injury [3-6]. Oropharyngeal and nasopharyngeal tract swabs need to be tested to confirm a clinical suspect of SARS-COV-2 infection [1]. Moreover, chest radiography and computed tomography (CT) scan complete the diagnostic approach to COVID-19 patients, usually showing groundglass opacities, sometimes associated with consolidative patterns [7-9]. In addition to these primary imaging methods, lung ultrasound (LUS) provides a timely bedside evaluation of COVID-19 patients, both in the primary assessment and during monitoring to adjust treatment plan [10].

\section{Case report}

A 37-year-old male, without known previous comorbidities, presented at the Emergency Department of Fondazione Poliambulanza Hospital (a medium-size general private hospital in Brescia, northern Italy), complaining fever and cough for 2 weeks. Moreover, he reported progressive dyspnea, limiting his activities of daily living. 
Peripheral blood examinations showed mild lymphopenia $(750 / \mathrm{mmc})$ and increased C-reactive protein $(10 \mathrm{mg} / \mathrm{L})$. Physical examination revealed a body temperature of $38{ }^{\circ} \mathrm{C}$, respiratory rate of 30 breaths per minute, and blood oxygen saturation of $90 \%$ on room air. Arterial blood gas test revealed moderate hypoxemia (pressure of arterial oxygen $-\mathrm{PaO} 2=50 \mathrm{mmHg}$ ) and mild hypocapnia $(\mathrm{PaCO} 2=30 \mathrm{mmHg})$. Nasopharyngeal swab specimen was collected for testing SARS-COV-2, and polymerase chain reaction revealed positive viral nuclear acid in the sample. A primary bedside lung ultrasound (LUS) assessment was immediately performed to provide a real-time estimate of COVID-19 lung involvement. The scan performed with convex array probe showed multifocal and confluent B-lines with thickening of the pleural line at the medium right field (Fig. 1) and a dynamic air bronchogram sign at the posterior homolateral lower field (Fig. 2). On the left side, the LUS showed no pleural sliding or lung point sign (Fig. 3). Chest X-ray confirmed a massive pneumothorax of the left lung and involvement of the right one (Fig. 4). A chest tube was immediately placed, and a subsequent CT scan confirmed the lung re-expansion and bilateral consolidations (Fig. 5). During the hospitalization, considering the long-lasting symptoms, high-flow nasal cannula oxygen therapy and steroid therapy without antiviral drugs were provided. A final chest X-ray before the discharge showed significant improvement, and the patient, until now, is asymptomatic with no need of therapy.

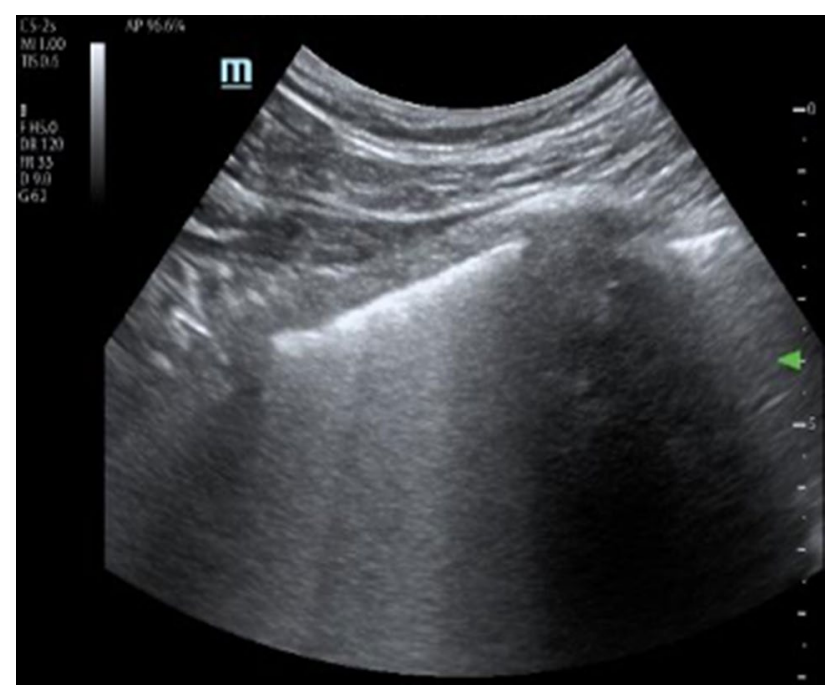

Fig. 1 Convex array probe showed multifocal and confluent B-lines with thickening of the pleural line in the medium field of the right side

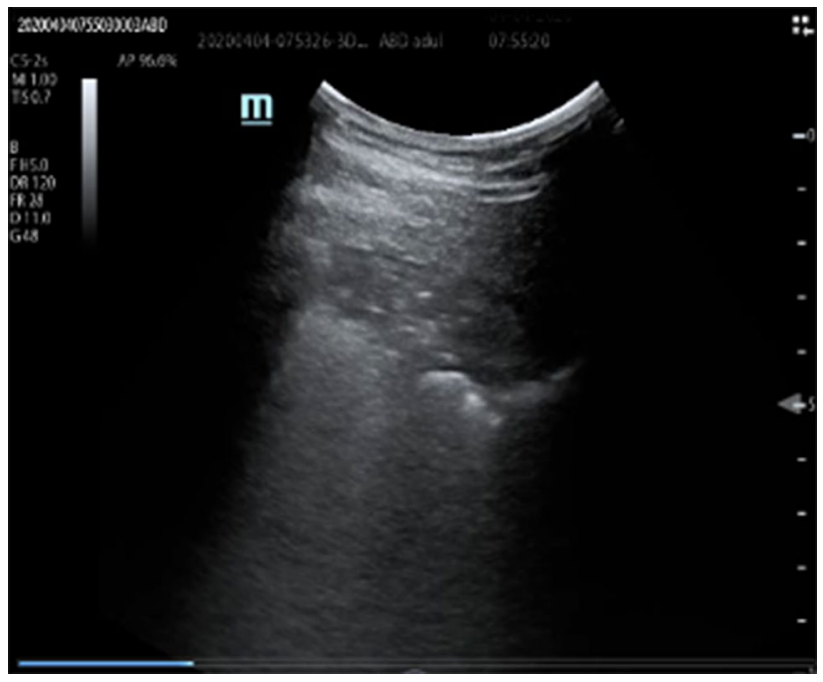

Fig. 2 Convex array probe showed consolidation with dynamic air bronchogram in inferior field of the right lung

\section{Discussion}

COVID-19 has been previously described as a highly infectious disease with several systemic complications $[2,5,6]$. Even though chest X-ray and CT scans are widely used in the primary instrumental assessment of COVID-19 patients [7-9], emerging evidence have explored the role of ultrasound in their diagnosis and treatment [10-12]. Frequent abnormal ultrasound imaging findings such as B-lines, consolidation areas, or alteration of the pleural line have been recently characterized [12]. On the other hand, ultrasound may produce a real-time and dynamic evaluation, even in

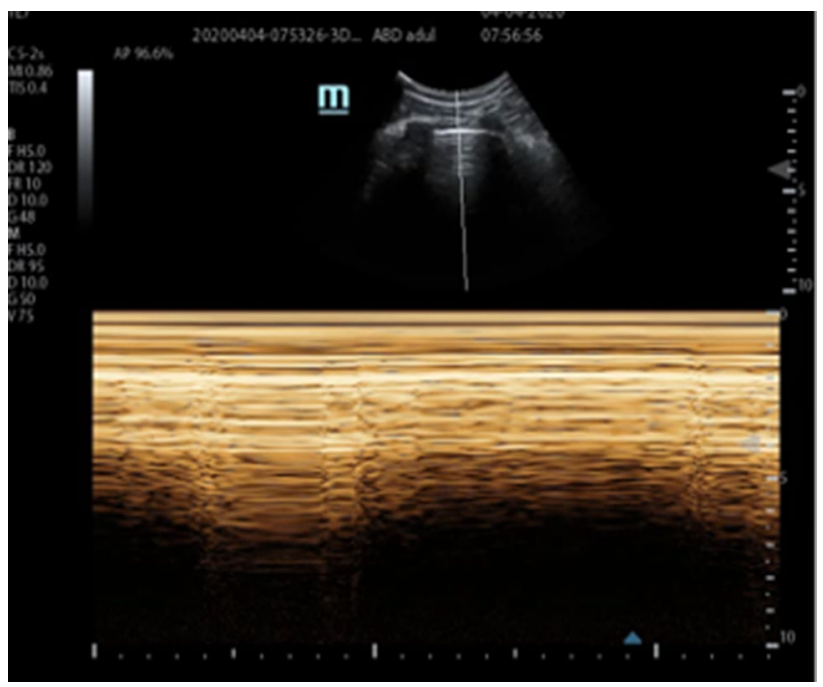

Fig. 3 Convex array probe showed the absence pleural sliding at the left lung with a "barcode sign" at the M-mode evaluation 


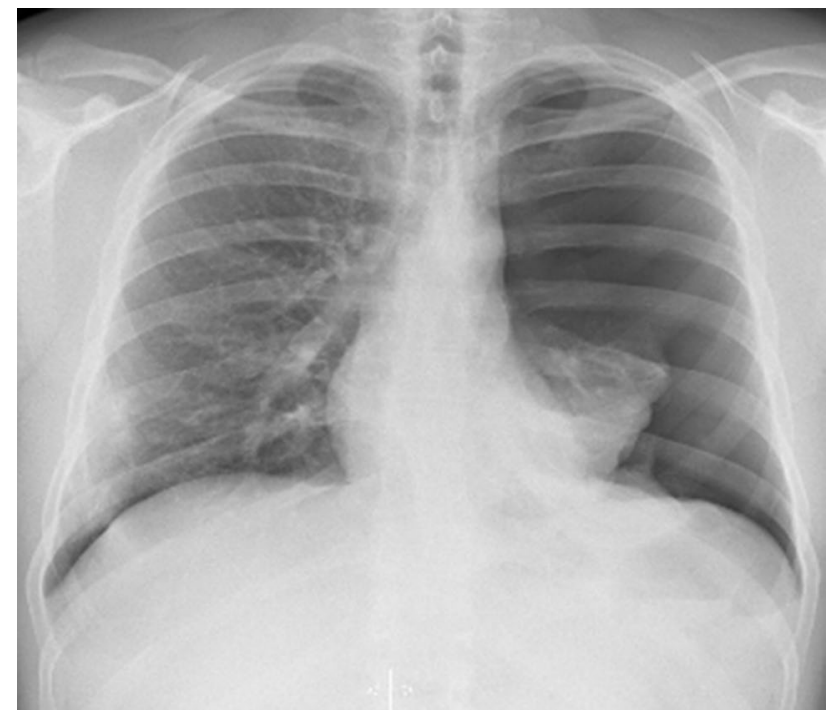

Fig. 4 Chest X-ray showed complete left pneumothorax and right interstitial involvement

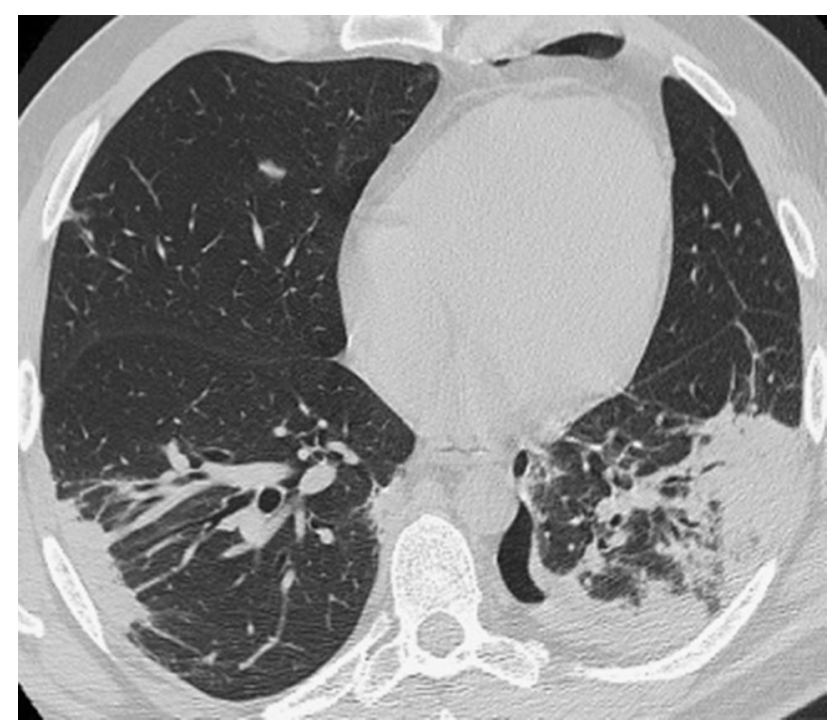

Fig. 5 CT-scan performed after chest tube placement showed bilateral consolidations, mainly at the posterior lower field of the left lung

cases with critical complications of severe COVID-19 pneumonia, such as pneumothorax. In addition, the appearance of these ultrasonic manifestations is not commonly closely related to the severity of interstitial lung involvement [13].

As described in the present report, COVID-19 infection, displaying its lung tropism, may be associated with multiple and diffuse lesions. Our data are consistent with those recently published by Sun et al. [13], who explored the outcome of a patient with mediastinal emphysema and pneumothorax. As detailed by Sun et al. pneumothorax could be produced as a consequence of a sudden increase of the alveolar

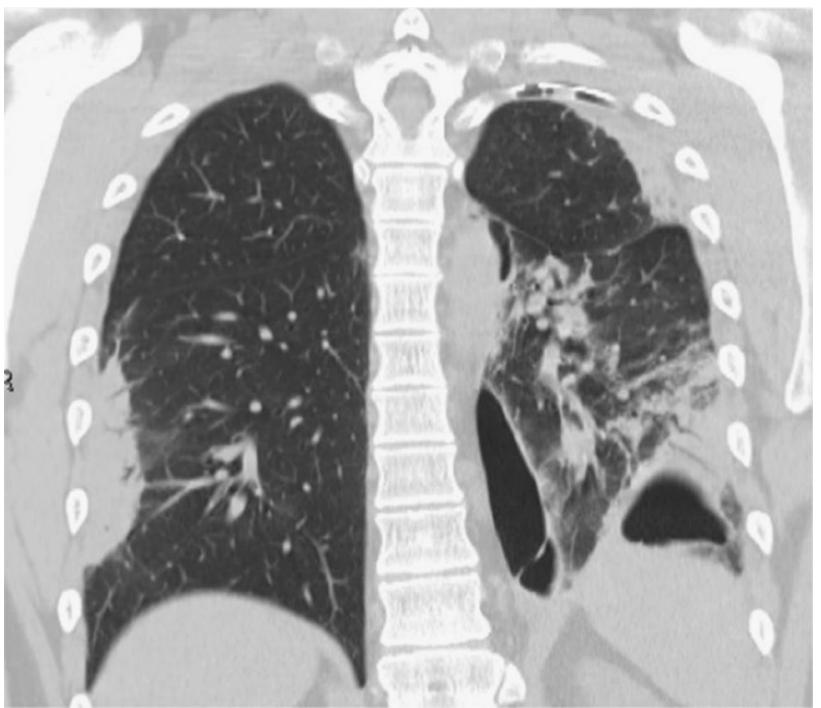

Fig. 6 CT-scan showed small pneumothorax after drainage and near the left alveolar consolidation

pressure in the pneumonic consolidations [13]. Lung compliance is high compared with other etiologies of ARDS, and the rate of barotrauma appears to be low, with only $2 \%$ developing pneumothorax compared with $25 \%$ of those with severe acute respiratory syndrome (SARS) $[5,6]$. Accordingly, the alveolar rupture of the patient described here was localized at the consolidated area, as revealed by the CT scan (Fig. 6). Along this line, lung ultrasonography could be performed at the patient's bedside, and it could be considered as a handy primary tool to quickly assess and subsequently treat the condition. The integration of ultrasound images with CT images may be effective in the activation of a comprehensive management plan. Growing evidence suggest the role of LUS in clinical decision-making and as a monitoring tool to assess patients during non-invasive ventilation, mostly when the presence of COVID-19-related complications such as pneumothorax may suddenly worsen the respiratory failure $[11,12]$.

In conclusion, lung ultrasound has emerged as a pointof-care diagnostic and monitoring tool to guide patient management. As shown in our report, ultrasound imaging findings can be useful to assess lung complications such as pneumothorax, even in patients without predisposing risk factors. Evidence of the clinical applications of LUS are of paramount importance.

Author contributions Conceptualization, L.C., P.T.; writing-original draft preparation, L.C., V.B., S.C., F.G; writing-review and editing, P.T., M.B., P.P.; supervision, P.T., V.B. All authors have read and agreed to the published version of the manuscript."

Funding This research received no external funding. 


\section{Compliance with ethical standards}

Conflicts of Interest The authors declare no conflict of interest.

Ethical approval All procedures performed in studies involving human participants were in accordance with the ethical standards of the institutional and/or national research committee and with the 1964 Helsinki declaration and its later amendments or comparable ethical standards.

Human and animal rights This article does not contain any studies with animals performed by any of the authors.

Informed consent Informed consent was obtained from all individual participants included in the study.

\section{References}

1. Guan WJ, Ni ZY, Hu Y, Liang WH, Ou CQ, He JX, Liu L, Shan H, Lei CL, Hui DSC, Du B, Li LJ, Zeng G, Yuen KY, Chen RC, Tang CL, Wang T, Chen PY, Xiang J, Li SY, Wang JL, Liang ZJ, Peng YX, Wei L, Liu Y, Hu YH, Peng P, Wang JM, Liu JY, Chen Z, Li G, Zheng ZJ, Qiu SQ, Luo J, Ye CJ, Zhu SY (2020) Clinical characteristics of coronavirus disease 2019 in China. New Engl J Med 382(18):1708-1720. https://doi.org/10.1056/NEJMoa2002 032

2. Wu Z, McGoogan JM (2020) Characteristics of and important lessons from the coronavirus disease 2019 (COVID-19) outbreak in China: summary of a report of 72314 cases from the Chinese center for disease control and prevention. JAMA. https://doi. org/10.1001/jama.2020.2648

3. Chen G, Wu D, Guo W, Cao Y, Huang D, Wang H, Wang T, Zhang X, Chen H, Yu H, Zhang X, Zhang M, Wu S, Song J, Chen T, Han M, Li S, Luo X, Zhao J (2020) Ning Q (2020) Clinical and immunologic features in severe and moderate coronavirus disease 2019. J Clin Invest 130(5):2620-2029. https://doi.org/10.1172/ JCI137244

4. Xu Z, Shi L, Wang Y, Zhang J, Huang L, Zhang C, Liu S, Zhao P, Liu H, Zhu L, Tai Y, Bai C, Gao T, Song J, Xia P, Dong J, Zhao J, Wang FS (2020) Pathological findings of COVID-19 associated with acute respiratory distress syndrome. Lancet Respir Med 8(4):420-422. https://doi.org/10.1016/S2213-2600(20)30076-X

5. Gomersall CD, Joynt GM, Lam P, Li T, Yap F, Lam D, Buckley TA, Sung JJ, Hui DS, Antonio GE, Ahuja AT, Leung P (2004)
Short-term outcome of critically ill patients with severe acute respiratory syndrome. Intensive Care Med 30(3):381-387. https ://doi.org/10.1007/s00134-003-2143-y

6. Yang X, Yu X, Xu J, Shu H, Xia J, Liu H, Wu Y, Zhang L, Yu Z, Fang M, Yu T, Wang Y, Pan S, Zou X, YuanShang SY (2020) Clinical course and outcomes of critically ill patients with SARSCoV-2 pneumonia in Wuhan, China: a single centered, retrospective, observational study. Lancet Respirat Med 8(5):475-481. https://doi.org/10.1016/S2213-2600(20)30079-5

7. Ye Z, Zhang Y, Wang Y, Huang Z, Song B (2020) Chest CT manifestations of new coronavirus disease 2019 (COVID-19): a pictorial review. Eur Radiol. https://doi.org/10.1007/s00330-02006801-0

8. Zhong Z, Xie X, Zhao W, Zheng C, Wang F, Liu J (2020) Chest CT for typical 2019-nCoV pneumonia: relationship to negative RT-PCR testing. Radiology. https://doi.org/10.1148/radiol.20202 00343

9. Zhao X, Liu B, Yu Y, Wang X, Du Y, Gu J, Wu X (2020) The characteristics and clinical value of chest $\mathrm{CT}$ images of novel coronavirus pneumonia. Clin Radiol 75(5):335-340. https://doi. org/10.1016/j.crad.2020.03.002

10. Vetrugno L, Bove T, Orso D, Barbariol F, Bassi F, Boero E, Ferrari G, Kong R (2020) Our Italian experience using lung ultrasound for identification, grading and serial follow-up of severity of lung involvement for management of patients with COVID19. Echocardiography 37(4):625-627. https://doi.org/10.1111/ echo. 14664

11. Sikachi R, Agrawal A (2020) Whole body point of care ultrasound for COVID-19: a multi-system approach to a multi-system disease. Anaesthesia. https://doi.org/10.1111/anae.15087

12. Sofia S, Boccatonda A, Montanari M, Spampinato M, D'ardes D, Cocco G, Accogli E, Cipollone F, Schiavone C (2020) Thoracic ultrasound and SARS-COVID-19: a pictorialessay. J Ultrasound. https://doi.org/10.1007/s40477-020-00458-7

13. Sun R, Liu H, Wang (2020) Mediastinal emphysema, giant bulla, and pneumothorax developed during the course of COVID19 pneumonia. Korean J Radiol 21(5):541-544. https://doi. org/10.3348/kjr.2020.0180

Publisher's Note Springer Nature remains neutral with regard to jurisdictional claims in published maps and institutional affiliations. 